\title{
CMEARTICLE
}

\section{Clinics in diagnostic imaging (196)}

Kheng Song Leow ${ }^{1}$, MD, FRCR, Poh Lye Paul See ${ }^{2}$, MBBS, FRCR
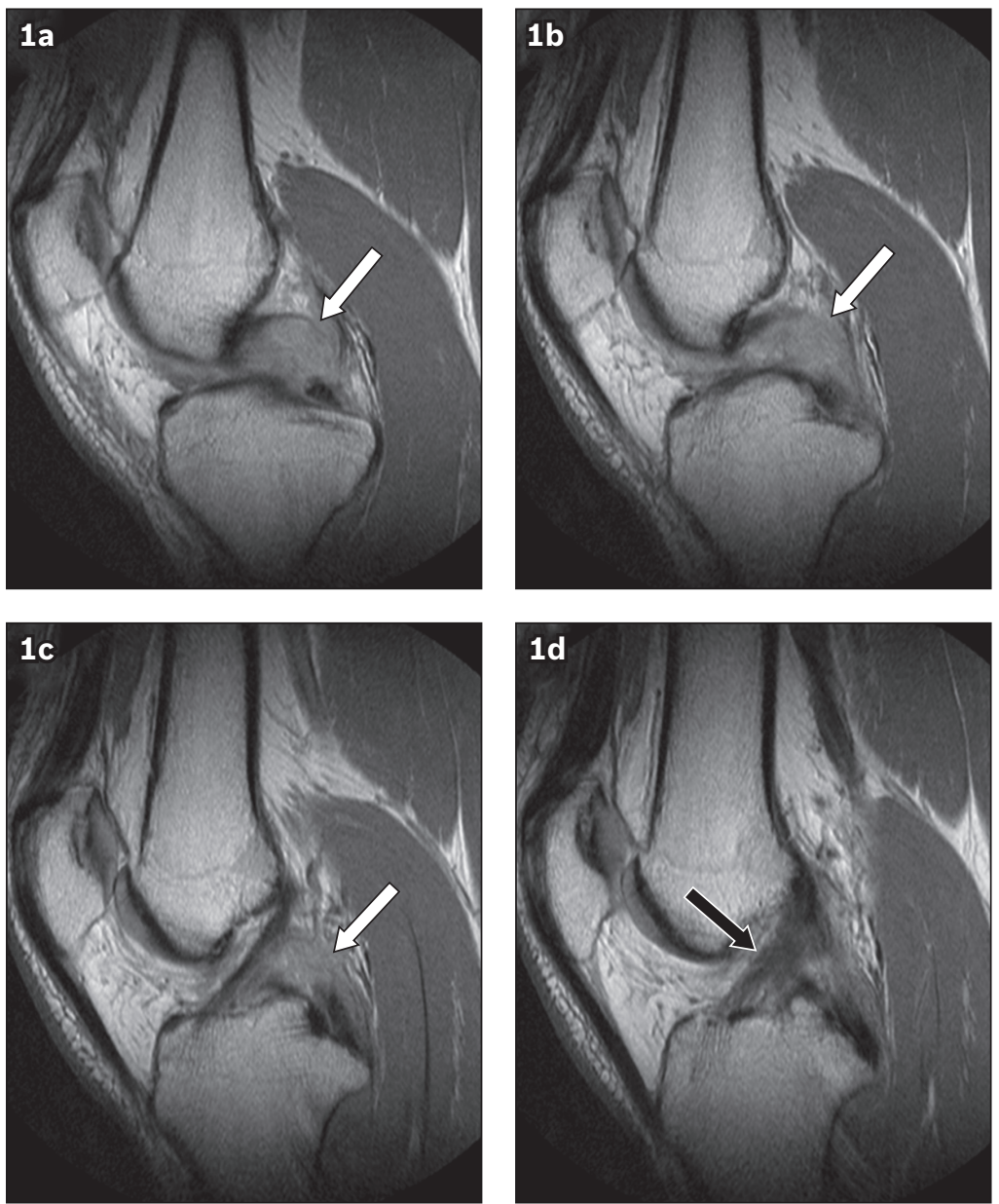

Fig. $1(a-d)$ Contiguous sagittal non-fat-suppressed proton density MR images of the left knee.

\section{CASE PRESENTATION}

A 21-year-old man presented with left knee pain and instability from an injury sustained in a road traffic accident three months prior. He had been riding a motorcycle when he self-skidded, landing on the left side of his body. Besides the left knee injury, he had also sustained fractures of the left little and middle fingers (not elaborated here). Physical examination of the left knee revealed a positive posterior drawer test. Anterior drawer and pivot tests were negative. Magnetic resonance (MR) imaging of the left knee was performed. What do the serial medial-tolateral sagittal images of the knee (Fig. 1) show? What is the diagnosis? 


\section{IMAGE INTERPRETATION}

Contiguous sagittal non-fat-suppressed proton density (PD) MR images of the knee show complete disruption of the posterior cruciate ligament $(\mathrm{PCL})$ at the mid portion (white arrows, Figs. 1a-c). The anterior cruciate ligament (black arrow, Fig. 1d) is not fully assessed, while the medial and lateral collateral ligaments are not seen and cannot be commented on. A review of the complete study showed these to be intact.

\section{DIAGNOSIS}

Complete PCL tear.

\section{CLINICAL COURSE}

The patient subsequently underwent $\mathrm{PCL}$ reconstruction with an allograft. Postoperatively, he was compliant with extensor musclestrengthening physiotherapy. Mild pain was experienced over the medial aspect of the knee joint, likely from the site of allograft harvesting, and was managed with oral analgesia.

\section{DISCUSSION}

The PCL is a dense, fibrous collagen band that originates from the lateral aspect of the medial femoral condyle to insert into the posterior intercondyloid (of PCL) fossa just inferior to the joint line. It is composed of two bundles, the anterolateral and posteromedial bundles, that are tightly bound together, giving it a uniformly low signal intensity on all MR pulse sequences. ${ }^{(1)}$ The $\mathrm{PCL}$ is nourished by the middle geniculate artery and innervated by branches of the tibial nerve. ${ }^{(2)}$

The PCL functions to restrict posterior translation of the tibia in relation to the femur. It also contributes to the restraint of internal rotation (with the knee in flexion of $90^{\circ}$ or more). ${ }^{(2)}$ In about $49 \%$ of knees, the anterior (Humphrey) and posterior (Wrisberg) meniscofemoral ligaments may be seen, both of which have been reported to contribute to posterior stability in PCLdeficient knees. ${ }^{(3)}$ Along with the anterior cruciate and medial and lateral collateral ligaments, the PCL contributes to overall knee stability and kinematics. ${ }^{(4)}$

Compared to injuries of the anterior cruciate ligament $(\mathrm{ACL})$, injuries afflicting the $\mathrm{PCL}$ are relatively uncommon. About 200,000 cases of ACL injuries are reported per year in the United States, while only approximately $3 \%$ of the injured knees have PCL tears. ${ }^{(5)}$ Three common mechanisms of injuries have been proposed: (a) posterior tibial translational force in a flexed knee, (b) exaggerated forceful hyperextension of the knee, or (c) knee rotation in combination with varus or valgus force. The two most common causes are motor vehicle accidents and sports injuries, accounting for about $57 \%$ of cases in total. ${ }^{(2)}$

The PCL is rarely injured in isolation. Instead, a PCL tear is often associated with other ligamental, meniscal and bony injuries: the $\mathrm{ACL}$ is involved in about $46 \%$ of cases, the medial collateral ligament in $31 \%$ of cases and the posterolateral corner complex in $62 \%$ of cases. ${ }^{(6)}$ Patterns of bone contusion may suggest the underlying mechanism of injury. For example, bone contusion in the anterior tibial plateau and posterior femoral condyle is commonly observed in dashboard injuries, while
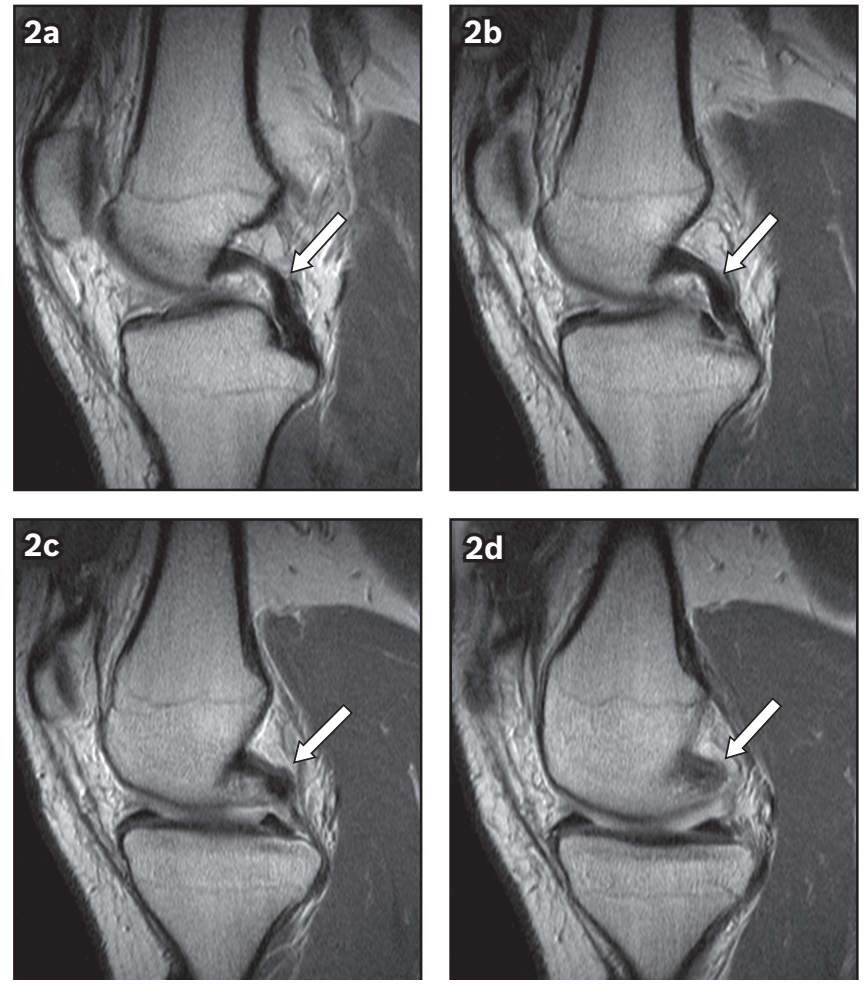

Fig. $2(a-d)$ Contiguous sagittal non-fat suppressed proton density MR images of the knee show the normal appearance of the posterior cruciate ligament (white arrows), from a lateral to medial direction.

bone oedema in the anterior aspects of both the tibial plateau and femoral condyle is suggestive of a hyperextension injury. ${ }^{(7)}$

A PCL injury is suggested by a classic history, such as posterior knee pain or instability, or positive findings on physical examination such as a posterior sag sign, positive posterior drawer test or positive quadriceps active test. ${ }^{(2)}$ However, the physical examination may be difficult or misleading due to pain, the presence of haemarthrosis, and other concurrent ligamentous injuries limiting assessment. ${ }^{(8)}$ In addition, when the ACL is intact, the PCL can be difficult to evaluate arthroscopically from the anterior approach. ${ }^{(9)}$ Thus, MR imaging can be valuable in bridging the diagnostic gap.

In the sagittal plane, a normal PCL is seen as a well-defined continuous band of low signal intensity (in all pulse sequences) in the intercondylar notch. It is lax and forms a gentle arc pointing posteroinferiorly with the knee being imaged in a position of partial extension. It measures no more than $7 \mathrm{~mm}$ in anteroposterior diameter in the sagittal images (Fig. 2). ${ }^{(8)}$ The use of a combination of both short-echo-time PD and long-echo-time T2-weighted sequences is encouraged to evaluate the PCL for tears with improved accuracy. ${ }^{(8)} \mathrm{PD}$ images are more sensitive for demonstrating abnormal signal intensity in the torn $\mathrm{PCL}$, but they tend to overestimate the PCL diameter and overdiagnose PCL tears due to their inherent limitation in differentiating abnormal signal intensity of the PCL from the surrounding oedematous soft tissues. ${ }^{(8)}$ T2-weighted sequences are useful to overcome this pitfall.

The PCL can be injured with varying degrees of severity, ranging from a sprain to a partial or complete tear. When the $\mathrm{PCL}$ measures $7 \mathrm{~mm}$ or greater, it is highly suggestive of a tear, with 

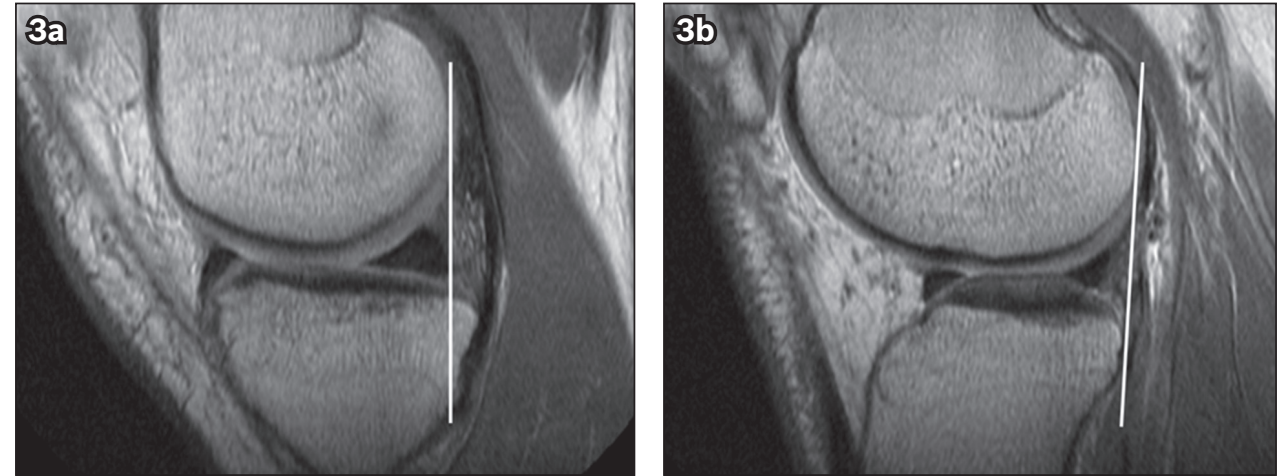

Fig. 3 Contiguous sagittal non-fat suppressed proton density MR images of the left knee of our patient shows the posterior tibial translation measuring about 4-5 mm more in the mid-medial compartment (vertical line in 3a) compared to the mid-lateral compartment (vertical line in 3b).
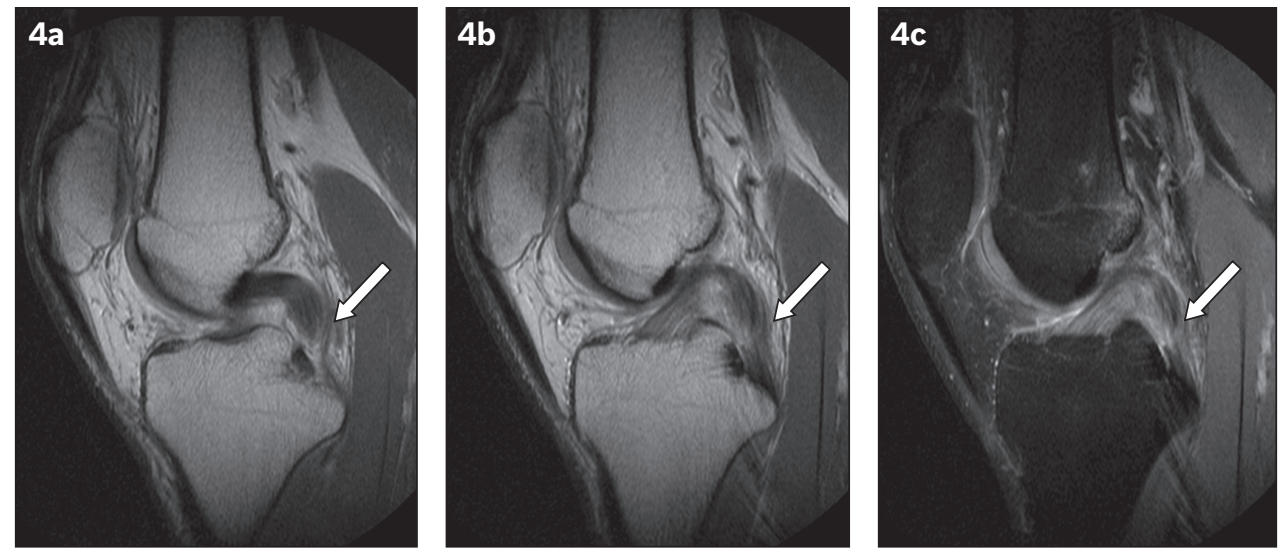

Fig. 4 (a-b) Contiguous sagittal proton density MR images through the intercondylar area show focal thickening and partial thickness fibre disruption in the distal portion of the posterior cruciate ligament (PCL) (arrows in a \& b), in keeping with a partial tear. (c) Sagittal T2-W MR image shows corresponding increased intrasubstance signal intensity in the PCL (arrow).
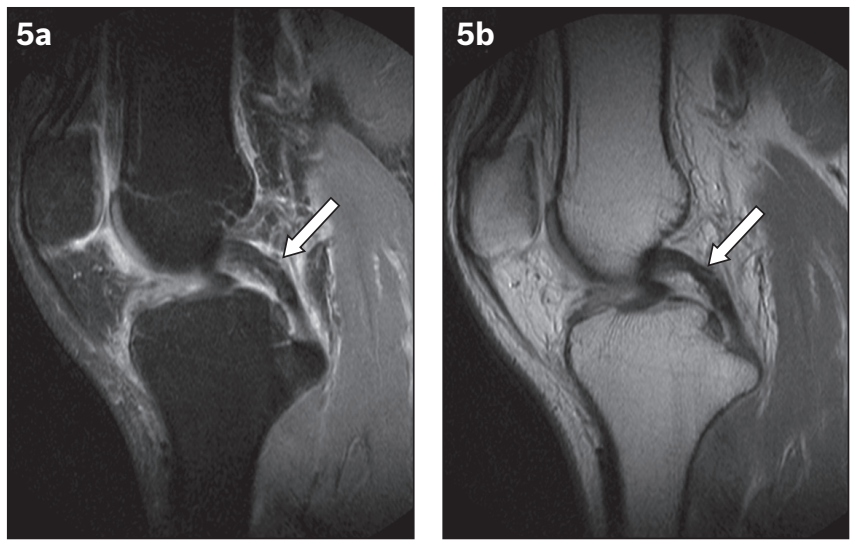

Fig. 5 (a) Sagittal fat-suppressed T2-W and (b) non-fat-suppressed proton density MR images show increased T2 signal intensity in the posterior cruciate ligament (PCL) (arrows), without obvious fibre interruption. The PCL measures less than $7 \mathrm{~mm}$ in anteroposterior diameter. Overall features suggest a sprained PCL.

a reported sensitivity of $94 \%$ and specificity of $92 \% .{ }^{(8)}$ Posterior tibial translation may serve as a valuable secondary sign of $\mathrm{PCL}$ tear, especially when it measures more than $+2.93 \mathrm{~mm}$ in the mid-medial compartment in relation to the mid-lateral compartment. ${ }^{(10)}$ This finding was observed in our patient (Fig. 3). A complete PCL tear is diagnosed when the PCL cannot be identified in the anatomical location or shows abnormal increased signal intensity without definable ligamentous fibres, ${ }^{(7)}$ which was also observed in our patient. When some of the
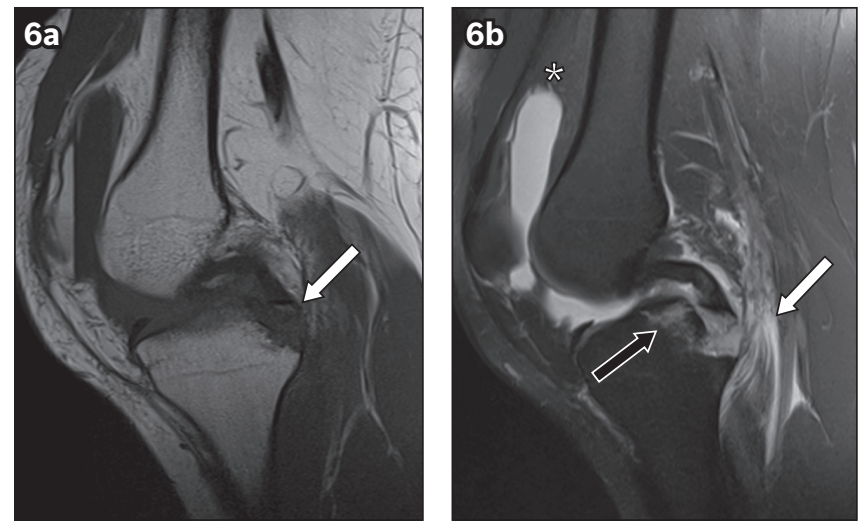

Fig. 6 (a) Sagittal T1-W MR image shows a small displaced bone fragment (arrow) with a signal intensity similar to that of bone marrow attached to the distal end of the posterior cruciate ligament (PCL), consistent with an avulsion fracture of the tibial attachment of the PCL. (b) Sagittal fat-suppressed T2-W MR image further demonstrates acute bone marrow oedema of the proximal tibia (black arrow) related to the aforementioned fracture, soft tissue oedema of the medial gastrocnemius muscle (white arrow) and moderate knee effusion with fat-fluid level in keeping with a lipohaemarthrosis (asterisk)

fibres are still intact, it is suggestive of a partial tear (Fig. 4). ${ }^{(7)}$ On the contrary, when the PCL shows increased T2-weighted intrasubstance signal intensity without obvious disruption of the ligamentous fibres, it is likely due to a sprain, ${ }^{(7)}$ as in the case in Fig. 5. A PCL injury may also involve the tibial attachment with an associated avulsion fracture, as shown in Fig. 6. The latter is one of the indications for surgery. ${ }^{(7)}$ 
Details on the treatment of PCL tears is beyond the scope of this article. Nonetheless, the management of PCL tears is generally dependent on two factors: (a) whether they are isolated or combined injuries (together with other ligament or meniscal tears) and (b) whether they are acute or chronic. ${ }^{(2)}$ An isolated PCL tear is usually managed conservatively, as it has an intrinsic ability to heal. The healing process usually occurs with the ligament in a lax position, hence the knee should be immobilised in full extension (for about 2-4 weeks), with bracing applied (to counteract the posterior tibial sagging) and subsequent quadriceps muscle strengthening exercises (to enhance the muscles that compensate for the posterior tibial translation). ${ }^{(2)}$

Other concomitant ligamentous or meniscal injuries, as well as persistent instability of the knee, are often an indication for surgical treatment. In the case of our patient, although he did not suffer other ligamentous or meniscal injuries, $\mathrm{PCL}$ reconstruction was performed in view of his persistent complaint of instability despite conservative treatment. Most techniques involve arthroscopy with either single- or double-bundle reconstruction, using autografts or allografts. Other fixation methods include the use of a trans-tibial tunnel or the tibial inlay technique. In brief, the single-bundle option aims to replace the anterolateral bundle, while double-bundle reconstruction attempts to restore normal knee kinematics by replacing both bundles. Autografts are harvested from the patellar (bone-patellar tendon-bone) or hamstring tendon, while allografts are often retrieved from the Achilles or quadriceps tendons. ${ }^{(2)}$

\section{CONCLUSION}

Recognition of the normal appearance of the PCL and spectrum of PCL injuries is useful for improved diagnosis of a PCL tear. Isolated acute PCL tears are usually managed conservatively. However, the active search for associated injuries should be emphasised, as their presence may upstage the patients for surgical management.
ABSTRACT A 21-year-old man presented with left knee pain and instability that persisted three months after a road traffic accident. Physical examination revealed a positive posterior drawer test. Anterior drawer and pivot tests were negative. Magnetic resonance imaging of the knee demonstrated a complete tear of the posterior cruciate ligament (PCL). Recognition of the normal and injured appearances of the PCL is useful to aid the reader in the detection and characterisation of $\mathrm{PCL}$ injuries. Isolated acute PCL tears are usually managed conservatively. However, an active search for associated injuries is essential, as their presence may upstage the patients for surgical management.

Keywords: knee joint, magnetic resonance imaging, pain, posterior cruciate ligament, rupture

\section{REFERENCES}

1. Vohra S, Arnold G, Doshi S, Marcantonio D. Normal MR imaging anatomy of the knee. Magn Reson Imaging Clin N Am 2011; 19:637-53.

2. Vaquero-Picado A, Rodríguez-Merchán EC. Isolated posterior cruciate ligament tears: an update of management. EFORT Open Rev 2017; 2:89-96.

3. Gupte CM, Bull AM, Thomas RD, Amis AA. The meniscofemoral ligaments: secondary restraints to the posterior drawer. Analysis of anteroposterior and rotary laxity in the intact and posterior-cruciate-deficient knee. J Bone Joint Surg Br 2003; 85:765-73.

4. Kennedy NI, Wijdicks CA, Goldsmith MT, et al. Kinematic analysis of the posterior cruciate ligament, part 1: the individual and collective function of the anterolateral and posteromedial bundles. Am J Sports Med 2013; 41:2828-38.

5. Morelli V, Bright C, Fields A. Ligamentous injuries of the knee: anterior cruciate, medial collateral, posterior cruciate, and posterolateral corner injuries. Prim Care 2013; 40:335-56.

6. Fanelli GC, Edson CJ. Posterior cruciate ligament injuries in trauma patients: Part II. Arthroscopy 1995; 11:526-9.

7. Sonin AH, Fitzgerald SW, Hoff FL, Friedman H, Bresler ME. MR imaging of the posterior cruciate ligament: normal, abnormal, and associated injury patterns. Radiographics 1995; 15:551-61.

8. Rodriguez W Jr, Vinson EN, Helms CA, Toth AP. MRI appearance of posterior cruciate ligament tears. AJR Am J Roentgenol 2008; 191:1031.

9. Gillquist J, Hagberg G, Oretorp N. Arthroscopy in acute injuries of the knee joint. Acta Orthop Scand 1977; 48:190-6.

10. Degnan AJ, Maldjian C, Adam RJ, Harner CD. Passive posterior tibial subluxation on routine knee MRI as a secondary sign of PCL tear. Radiol Res Pract 2014; 2014:715439. 


\section{SINGAPORE MEDICAL COUNCIL CATEGORY 3B CME PROGRAMME} (Code SMJ 201906B)

Question 1. Regarding the incidence, clinical presentation and mechanism of injury of posterior cruciate ligament $(\mathrm{PCL})$ tear:

(a) PCL injury is relatively uncommon compared to its counterpart, the anterior cruciate ligament $(\mathrm{ACL})$ injury.

(b) The most common aetiologies are motor vehicle accidents and sports injuries.

(c) Proposed mechanisms of injury include exaggerated forceful hyperflexion of the knee, posterior tibial translational force in a flexed knee, and knee rotation in combination with varus or valgus force.

(d) Posterior drawer or positive quadriceps active tests are often performed in patients with suspected PCL tears.

Question 2. Regarding the anatomy and functions of the PCL:

(a) The PCL is a dense, fibrous collagen band that originates from the lateral aspect of the medial femoral condyle to insert into the posterior intercondyloid fossa.

(b) The PCL is composed of anteromedial and posterolateral bundles that are tightly bound together.

(c) The PCL functions only to restrict the posterior translation of the tibia in relation to the femur.

(d) Other ligamentous structures of the knee, namely the ACL, collateral ligaments and meniscofemoral ligaments, contribute to the overall stability of the knee joint.

Question 3. Regarding the imaging of PCL injuries:

(a) Imaging, namely magnetic resonance $(M R)$ imaging, has no role in the management of PCL injury.

(b) Normal PCL is a uniformly low signal ligamentous structure in the knee joint on all MR pulse sequences.

(c) It is normal for the PCL to appear lax when MR imaging of the knee is performed in partial extension.

(d) Proton density-weighted sequence alone is sufficient to accurately characterise a PCL tear.

Question 4. Regarding the imaging features of PCL injury:

(a) When the PCL is no longer seen in the anatomical location, it is suggestive of a partial tear.

(b) A PCL measuring $7 \mathrm{~mm}$ or more with incomplete fibre disruption is highly suggestive of a partial tear.

(c) When the PCL shows increased signal on T2-weighted image without ligamentous fibre disruption, it is likely to be sprained.

(d) An avulsion fracture involving the tibial attachment of the PCL is part of the spectrum of PCL injuries.

Question 5. Regarding the management of a PCL tear:

(a) Surgical treatment is often indicated in patients with acute complete PCL tear and other associated ligamentous or meniscal injuries.

(b) PCL reconstruction can be performed using autografts or allografts.

(c) In PCL reconstruction, the single-bundle technique aims to replace the anterolateral bundle, while double-bundle reconstruction attempts to restore normal knee kinematics by replacing both bundles.

(d) Autografts are often harvested from the Achilles tendons.

True

False

$\square$

$\square$

$\square$

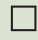

$\square \quad \square$

$\square$

$\square$

$\square$

\section{Doctor's particulars:}

Name in full:

Specialty:
MCR no:

Email:

\footnotetext{
SUBMISSION INSTRUCTIONS:

Visit the SMJ website: http://www.smj.org.sg/current-issue and select the appropriate quiz. You will be redirected to the SMA login page.

For SMA member: (1) Log in with your username and password (if you do not know your password, please click on 'Forgot your password?'). (2) Select your answers for each quiz and click 'Submit'.

For non-SMA member: : (1) Create an SMJ CME account, or log in with your SMJ CME username and password (for returning users). (2) Make payment of SGD 21.40 (inclusive of $7 \%$ GST) via PayPal to access this month's quizzes. (3) Select your answers for each quiz and click 'Submit'.

RESULTS:

(1) Answers will be published online in the SMJ August 2019 issue. (2) The MCR numbers of successful candidates will be posted online at the SMJ website by 8 August 2019. (3) Passing mark is $60 \%$. No mark will be deducted for incorrect answers. (4) The SMJ editorial office will submit the list of successful candidates to the Singapore Medical Council. (5) One CME point is awarded for successful candidates. (6) SMC credits CME points according to the month of publication of the CME article (i.e. points awarded for a quiz published in the December 2017 issue will be credited for the month of December 2017, even if the deadline is in January 2018).

Deadline for submission (June 2019 SMJ 3B CME programme): 12 noon, 1 August 2019.
} 